Objectives: Use CRISPR-Cas9, to perturb the COG6 intronic enhancer region and measure the downstream effect on the expression of FOXO1.

Methods: We utilised a modified form of the Cas9 enzyme, dead Cas9 (dCas9), that can precisely target DNA, but will not induce a cut. Using the dCas9 attached to either enhancers (p300) or repressors (KRAB) of expression we investigated how perturbation of the enhancer intronic of COG6 changed the expression of FOXO1.

We designed 3 guides across the COG6 enhancer, and transduced a cell line (HEK293) using a lentiviral dCas9 CRISPR system, with either dCas9-KRAB or dCas9-p300 and each of the three guides. We cultured the cells until $70 \%-80 \%$ confluent, GFP sorted the cells and then extracted RNA. A quantitative PCR was performed (QuantStudio) for both COG6 and FOXO1 gene transcript expression and normalised to housekeeping genes.

Results: Up to $90 \%$ of HEK cells were tranduced with the dCas9 enzyme and guide, and these were sorted by FACS using GFP to sort the top $60 \%$. The 3 guides gave consistently increased levels of FOXO1 expression with the dCas9$\mathrm{p} 300$, compared to both control and dCas9-KRAB $(p=0.02)$. This was particular evident for guide 3 , with a $40 \%$ increase (p300) and $10 \%$ decrease (KRAB) of FOXO1 expression observed. Expression of COG6 was also perturbed, but in a less consistent manner, with both increase and decrease expression for KRAB and $\mathrm{p} 300$.

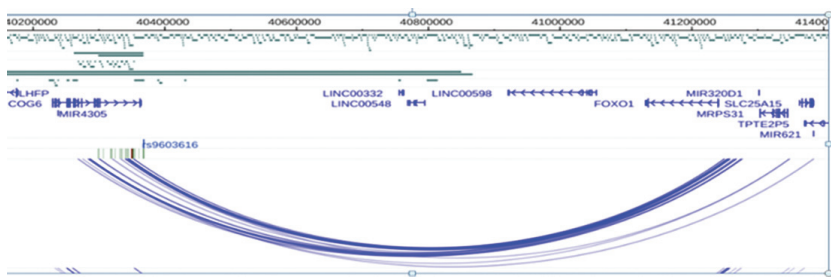

Conclusions: Over $90 \%$ of HEK cells were tranduced with the dCas9 enzyme and guide, and these were sorted by FACS using GFP to sort the top $60 \%$. The 3 guides gave consistently increased levels of FOXO1 expression with the dCas9$\mathrm{p} 300$, compared to both control and dCas9-KRAB $(p=0.02)$. This was particular evident for guide 3 , with a $40 \%$ increase (p300) and $10 \%$ decrease (KRAB) of FOXO1 expression observed. Expression of COG6 was also perturbed, but in a less consistent manner, with both increase and decrease expression for KRAB and $\mathrm{p} 300$.

REFERENCE:

[1] Martin $\mathrm{P}$, et al. Capture Hi-C reveals novel candidate genes and complex long-range interactions with related autoimmune risk loci. Nat Commun 2015 Nov 30.

Disclosure of Interest: None declared

DOI: 10.1136/annrheumdis-2018-eular.3946

\section{OP0289 INTEGRATION OF CHROMATIN CONFORMATION, TRANSCRIPTOME AND GENOME-WIDE LANDSCAPE OF BRD2 AND BRD4 BINDING MOTIFS IDENTIFIES MECHANISMS OF BET INHIBITOR ACTION IN RHEUMATOID ARTHRITIS SYNOVIAL FIBROBLASTS}

S. Nagpal ${ }^{1}$, V. Krishna ${ }^{2}$, X. Yin ${ }^{1}$, D. Pocalyko ${ }^{2}$, A. Walsh ${ }^{1}$, K. Bachman ${ }^{2}$, I. Anderson ${ }^{1}$, L. Madakamutil ${ }^{1} .{ }^{1} /$ mmunology; ${ }^{2}$ Discovery Sciences, Janssen Research, Spring House, USA

Background: Rheumatoid arthritis $(\mathrm{RA})$ is a chronic autoimmune disease characterised by infiltration of immune cells into the synovium and hyperplasia of the synovial lining, resulting in the formation of pannus that degrades cartilage and bone. Fibroblast-like synoviocytes (FLS) are the main cell types of the rheumatoid synovium and possess phenotypic and molecular characteristics of transformed cells. JQ1, an inhibitor of the bromodomain and extra terminal domain (BET) family that includes BRD2, BRD3, BRD4 and BRDt has shown efficacy in vitro on RAFLS proliferation and in vivo in a murine model of arthritis.

Objectives: We sought to elucidate the mechanism of action of BET proteins in FLS biology and determine the potential therapeutic utility of targeting BRD2/ BRD4 for RA disease treatment and interception.
Methods: To understand the mechanism of JQ1 action, we subjected JQ1treated RA-FLS to transcriptional profiling by RNA-Seq and determined BRD2 and BRD4 cistromes by identifying global BRD2/BRD4 chromatin binding sites by ChIP-Seq. In addition, Assay for Transposable Accessible Chromatin by high throughput Sequencing (ATAC-Seq) was employed to identify open and closed regions of chromatin in JQ1-treated RA-FLS.

Results: We demonstrate that the active isomer of JQ1 but not its inactive isome inhibits IL-1b-induced RA-FLS activation and proliferation. Through an integrated analysis of RNA-Seq, ATAC-Seq to profile changes in chromatin accessibility and ChIP-Seq of BRD2/4 and Pol2 proteins, we found that JQ1 inhibited multiple key inflammatory pathways, and altered the genome wide occupancy of crucial transcription factors involved in inflammatory signalling. Specifically, JQ1 treatment resulted in reduced occupancy of both BRD2 and BRD4 in approximately 2000 regions genome-wide, and a loss of Pol2 occupancy in approximately 600 genomic regions. Collectively we found that 105 genes had altered occupancy in all three proteins (BRD2, BRD4 and Pol2) and were also differentially expressed. Most prominently, JQ1 resulted in down regulation of IL6, IL8, p38 MAP kinase and HMGB1/TLR4 signalling pathways. In addition, we have identified BRD2 BRD4 super-enhancer genes and demonstrate that JQ1 altered BRD2/BRD4 occupancy in the IL6 and IL8 super-enhancer regions, and significantly downregulated IL6, IL8, TLR4 and IL1b expression.

Conclusions: Our results suggest pleiotropic effects of JQ1 on pathways that have been individually targeted and shown to be efficacious for the treatment of RA. These studies provide a strong rationale for targeting of BRD2/BRD4 in RA. Disclosure of Interest: S. Nagpal Shareholder of: Johnson and Johnson, Grant/ research support from: Johnson and Johnson, Employee of: Johnson and Johnson, V. Krishna Shareholder of: Johnson and Johnson, Grant/research support from: Johnson and Johnson, Employee of: Johnson and Johnson, X. Yin Shareholder of: Johnson and Johnson, Grant/research support from: Johnson and Johnson, Employee of: Johnson and Johnson, D. Pocalyko Shareholder of: Johnson and Johnson, Grant/research support from: Johnson and Johnson, Employee of: Johnson and Johnson, A. Walsh Shareholder of: Johnson and Johnson, Grant/ research support from: Johnson and Johnson, Employee of: Johnson and Johnson, K. Bachman Shareholder of: Johnson and Johnson, Grant/research support from: Johnson and Johnson, Employee of: Johnson and Johnson, I. Anderson Shareholder of: Johnson and Johnson, Grant/research support from: Johnson and Johnson, Employee of: Johnson and Johnson, L. Madakamutil Shareholder of: Johnson and Johnson, Grant/research support from: Johnson and Johnson, Employee of: Johnson and Johnson

DOI: 10.1136/annrheumdis-2018-eular.7388

FRIDAY, 15 JUNE 2018

\section{PARE abstract session}

\section{OP0290-PARE KEEP AN EYE OUT FOR INVISIBLE PAINS - A DANISH CAMPAIGN}

\section{C.R. Ziegler. Gigtforeningen, Gentofte, Denmark}

Background: How does it feel to live with a RMD and have chronic pain? How do you explain pain caused by RMD to your friends and family? How do you make others understand how pain affects your daily life? The communication department of the Danish Rheumatism Association needed to convey living with invisible pains, because it is of such importance to people with RMDs. Based on interviews with the users (people with RMDs) we found the best way of making others understand was to create a film, which illustrated living with invisible pain

Objectives: We wanted to increase the general understanding of RMDs and living with invisible pain in society. The secondary objective was to brand awareness of the Danish Rheumatism Association and to generate leads for further processing, e.g. signing up for membership.

Methods: Based on a few interviews with users and several posts from Social Media we focused on making invisible pain visible. By choosing the media of a film we could show what the users found difficult putting into words. The film had to be short to make it possible to share, e.g. on Facebook, and by choosing a film without speak we avoided language barriers. Through adverts we retargeted visitors to our website and generated more members of the association.

Results: We launched the film on World Arthritis Day 2017 In 311/2 weeks the film reached 1 million users and more than 1000 comments on Facebook, and the number of followers went up by 2000 . The total impact of the film will be presented in June 2018. 